\title{
External dacryocystorhinostomy in patients with granulomatous polyangiitis
}

\author{
Christopher M. Stewart ${ }^{1} \cdot$ Geoffrey E. Rose ${ }^{1}$
}

Received: 26 March 2019 / Revised: 21 June 2019 / Accepted: 13 August 2019 / Published online: 4 November 2019

(c) The Author(s), under exclusive licence to The Royal College of Ophthalmologists 2019

\begin{abstract}
Aim To review the outcome for primary or revisional external dacryocystorhinostomy in patients with granulomatous polyangiitis.

Patients and methods Retrospective case-note review to include age at presentation, duration of prior GPA, immunosuppression at time of surgery, nature of first lacrimal surgery, and symptomatic control.

Results Forty-eight patients (25 female; 52\%) presented at an average age of 50.5 years and $7(15 \%)$ had prior lacrimal surgery on one $(5 / 7 ; 71 \%)$ or both sides. The duration of systemic GPA varied, with $40 \%$ having disease for up to 3 years prior to lacrimal referral, and 41/48 (85\%) being on systemic immunosuppression. Forty-eight patients had 71 affected drainage pathways-having symptoms for an average of 22 months (range $<1$ month to 7 years). Surgery was undertaken on 70/71 systems - 62/70 primary DCRs (20 unilateral; 8 simultaneous bilateral; 13 sequential bilateral). Of nine systems with persistent symptoms after prior surgery, eight underwent revisional surgery (six unilateral; one sequential bilateral). With a follow-up of 2.5 years (range 3 months to 14 years), volume symptoms were cured in all 70 cases. Flow symptoms were controlled in 58/62 (94\%) systems after primary DCR, and all eight after revisional surgery. Of four sides with persistent epiphora, one was cured with revisional DCR and three with placement of Jones' canalicular bypass tubes.

Conclusion Mucoid discharge, dacryocystitis and recurrent conjunctivitis were cured in all patients with GPA after either primary and revisional surgery. Continued epiphora was controlled in most patients with additional closed procedures.
\end{abstract}

\section{Introduction}

Granulomatous polyangiitis (GPA; formerly Wegener's granulomatosis) is a systemic vasculitis that mainly affects the respiratory tract and kidneys, and may cause nasolacrimal duct stenosis as a result of adjacent extensive destruction and scarring of the nasal cavity (Fig. 1) [1]. Published outcomes for lacrimal drainage surgery in such patients are limited to small series, from multiple centres and using various techniques [1-5].

We present a retrospective review of the outcomes for 70 external dacryocystorhinostomies (DCRs) performed in 48 patients with systemic GPA.

Geoffrey E. Rose

geoff.rose1@nhs.net

1 Adnexal Service, Moorfields Eye Hospital, London EC1V 2PD, UK

\section{Patients and methods}

Patients with GPA undergoing external DCR under the care of a single surgeon (GER) between 1991 and 2017 were identified from records at Moorfields Eye Hospital, and data collected through a retrospective review of clinical casenotes. Patient details included age at presentation for lacrimal surgery, duration of prior GPA, immunosuppression at the time of surgery, the nature of first lacrimal surgery, and symptomatic control after surgery. For those with residual postoperative symptoms, the nature of any further lacrimal drainage procedures was also noted.

External DCR was consultant-performed or consultantled, using a uniform technique with a large osteotomy, suturing of posterior and anterior mucosal flaps, and silicone intubation [6]. All surgery was done at a time when the systemic disease was thought to be quiescent but, because GPA can spread to involve the orbit after DCR [7], a 2-3 week tapering dose of oral prednisolone was prescribed in most patients-in addition to their usual systemic immunosuppression. All patients received steroid-antibiotic 
Fig. 1 Typical nasal endoscopic appearance of patients with chronic sino-nasal granulomatous polyangiitis, showing a loss of nasal structures with extensive, thick crusting and haemorrhagic and scarred underlying mucosa, and b widespread scarring with a large nasal septal perforation

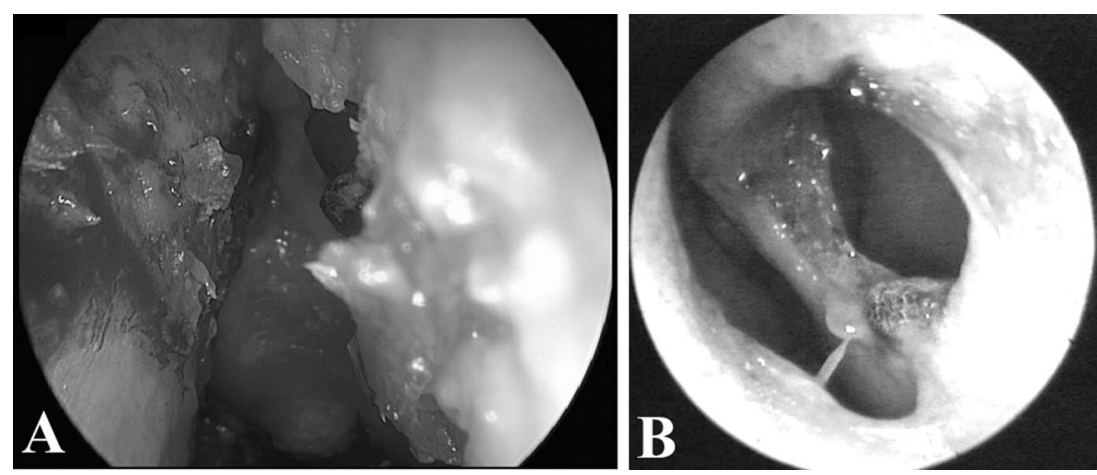

eyedrops for about a month, but no nasal medication or douching was used. Sutures were removed at 7-10 days, and intubation removed at 4-6 weeks after surgery. "Success" was defined as resolution of volume (mucoid discharge, dacryocystitis or recurrent conjunctivitis) and flow (epiphora) symptoms [8].

Although external DCR is theoretically the same as that for a normal patient, the heavily-scarred nasal space with GPA often requires modified techniques. In particular, GPA progressively obliterates the nasal cavity from antero-superiorly to postero-inferiorly and, in advanced cases, there can be no air-space alongside most of the lacrimal sac: when this is the case, it is vital to create a very large and low osteotomy extending well down into the upper nasolacrimal duct, thereby allowing the-often massively dilated-sac and upper duct to be sutured widely open; if this area is not widely opened, there is a tendency to get secondary loculation of the unopenable upper sac that can then drain retrogradely onto the eye [2] or a draining cutaneous fistula. In GPA the nasal mucosa can be extremely thick-sometimes $>1 \mathrm{~cm}$-and thinning of the nasal flaps (from the periosteal surface) may be required to give the flexibility needed to create a sutured anastomosis; failure to achieve a wide-open marsupialization of these sacs runs a major risk of both sump syndrome and cutaneous fistula formation-these being not uncommon with endonasal or inadequate external DCR.

The study received Institutional Research Board Approval (\#CA17/AD/13).

\section{Results}

Forty-eight patients ( 25 female; $52 \%$ ) were first diagnosed with GPA at 44.5 years of age (median 44; range 7-86). Eight patients (17\%) had GPA for less than a year, $40 \%$ for 1-3 years, $29 \%$ for $4-10$ years, and $14 \%$ for more than a decade-this being multi-system disease in 20/48 (42\%) and solely sino-nasal disease in 19/48 (40\%). All but seven patients $(85 \%)$ were taking systemic immunosuppression, with azathioprine and prednisolone being the commonest regime (14/41 patients; 34\%). The average age at referral was 50.5 years (median 54.5; range 13-88 years), symptoms were present for an average of 22 months (median 12; range $<1$ month to 7 years), and were bilateral in 23/48 (48\%). Epiphora was present in all 71 affected systems, and dacryocystitis occurred in 22/25 (88\%) patients with unilateral disease and 21/23 (91\%) with bilateral disease.

Nine systems (in seven patients) were referred for failed DCR performed, on average, 6 years before referral (median 2; range 1-15 years) — with all having persistent volume and flow symptoms. Four of these seven patients (57\%) had recurrent symptoms within a year of primary surgery, whereas the others had late $(>10$ year) recurrence.

A total of 62 primary and 8 revisional DCRs were performed at an average age of 51.6 years (median 56; range 14-88). The primary DCRs comprised eight simultaneous bilateral, 13 staged bilateral and 20 unilateral. Of the revisional DCRs, six were performed as unilateral surgery and one was sequential bilateral; one patient, referred with failed bilateral procedures, chose only to undergo unilateral revisional DCR. The lacrimal sac mucosa in these patients was often extremely thick and inflamed, due to the very longstanding lacrimal sac pyocoeles. Patients having revisional DCR usually had more intranasal scarring and adhesions, together with extensive fibrosis at the prior osteotomy - this necessitating a large osteotomy and extensive resection of fibrous tissue, to create a wide open soft-tissue mucosal anastomosis. Although most patients had widespread intranasal scarring, chronic mucosal inflammation and marked crusting both before and after DCR, there were no recorded operative or postoperative complications.

Outcome data was available for all patients to a mean follow-up of 2.5 years (median 9 months; range 3 months-14 years), with volume symptoms-viscous ocular discharge and dacryocystitis-being cured after all 70 procedures (whether primary DCR or revisional). Although there was early resolution of epiphora after all procedures, it recurred at 2, 3 and 10 years in three patients (after four primary DCRs) - all of these patients being on long-term systemic immunosuppression. Recurrent epiphora was cured in one patient after open revisional DCR (performed 2 years after the original procedure); the two other patients (three systems) underwent 
unsuccessful endonasal-guided Sisler transcanalicular trephination for extensive scarring of the common canaliculi, prior to successful symptom control with placement of Lester Jones tubes.

\section{Discussion}

Nasolacrimal duct obstruction is seen in $7 \%$ patients with GPA, this possibly increasing with improved patient survival [1, 3]. Our patient demographics have not changed significantly since an early small paper from Moorfields [2] (some of these patients included in the current series) but, in comparison with other publications [1, 4, 5], our current cohort is rather older at referral. Asymptomatic patients were usually discharged at 3 months after surgery, but our mean follow-up of 2.5 years (median 9 months; range 3 months-14 years) was similar to another case-series [5], but slightly less than that of two others $[1,4]$. As our study suggests recurrent epiphora may occur after some years without symptoms, it is possible that some late failures are missed in our follow-up; however, it is likely that the number of such "missed" patients is low, as our referral pattern (to a specialist unit within the prime UK eye hospital) would tend to return such patients to our care.

Whether for primary or revisional surgery, our DCR technique, using a large osteotomy and sutured posterior and anterior mucosal anastomoses [6], would appear to give complete cure for volume symptoms [8] (with no further dacryocystitis or viscous ocular discharge) in this group, despite often having extremely scarred nasal spaces (Fig. 1). Flow symptoms [8] recurred in 4/62 primary DCRs, with recurrence at 2, 3 and 10 years after surgery: of these four with recurrent flow symptoms, one was cured after revisional DCR and three had symptom control with Jones' tube placement. Using a similar external DCR technique with sutured anterior and posterior flaps, but a smaller $(10 \mathrm{~mm} \times 10 \mathrm{~mm})$ osteotomy, Naeser reports complete cure in eight patients [5], whereas it is unclear whether both anterior and/or posterior flaps were created and sutured in other papers [1, 4]. Some authors [1, 3] suggest that external DCR may result in excessive inflammation and fistula formation but, with the short-term use of extra postoperative oral steroids (a practice started after a patient developed orbital spread of GPA; [7] patient excluded from present study), we have not had any such complications. Although nasal scarring is known to cause DCR failure [1], some authors suggest that the limited size of endonasal osteotomy (in their four cases) was unimportant in the face of soft-tissue scarring [3]. We would disagree with this sentiment: despite heavily scarred and chronically inflamed nasal spaces, none of our patients had phimosis of the sutured anastomosis between the sac and nasal mucosae, and all of our late failures (4/62 patients after primary DCR) were due to adherence of the Valve of Rosenmuller over the internal end of the common canaliculus.

Most of our patients $(85 \%)$ were on regular systemic immunosuppression at the time of DCR, and all received a 2-3 week weaning regime of oral prednisolone after surgery - this being without major side-effects. Likewise, the literature suggests good results with continued perioperative immunosuppression [1, 2, 4, 5], and some authors report success without prescribing extra postoperative immunosuppression [4].

Revisional DCR in patients with GPA has been hardly reported, with $75 \%$ (3/4 patients) success [1, 2, 4]. Our complete cure of volume symptoms in $8 / 8$ revisional cases suggests an excellent outlook with wide and sutured marsupialization of the sac, even when there is markedly narrowed nasal space due to heavy scarring. All our patients having revisional surgery were on maintenance therapy, with all of this cohort receiving a short course of extra postoperative corticosteroids.

\section{Summary}

\section{What was known before}

- Granulomatous polyangiitis can cause gross scarring, or even occlusion, of the nasal space.

- The scarring of the nasal space leads to lacrimal drainage obstruction.

- Many small reports of lacrimal drainage surgery suggest a relatively poor outcome in these patients.

\section{What this study adds}

- The largest single-centre study of lacrimal drainage surgery in patients with granulomatous polyangiitis.

- Volume symptoms and signs can be cured with external dacryocystorhinostomy, either primary or revisional.

- Epiphora persists in a minority and, with most of such patients, the residual flow symptoms can be cured with a secondary closed procedure-such as placement of a canalicular bypass tube.

Acknowledgements Professor Geoffrey Rose receives some funding from the National Institute of Health Research (NIHR) Biomedical Research Centre at Moorfields Eye Hospital NHS Foundation Trust and UCL Institute of Ophthalmology.

\section{Compliance with ethical standards}

Conflict of interest The authors declare that they have no conflict of interest. 
Publisher's note Springer Nature remains neutral with regard to jurisdictional claims in published maps and institutional affiliations.

\section{References}

1. Hardwig PW, Bartley GB, Garrity JA. Surgical management of nasolacrimal duct obstruction in patients with Wegener's granulomatosis. Ophthalmology. 1992;99:133-9.

2. Kwan AS, Rose GE. Lacrimal drainage surgery in Wegener's granulomatosis. Br J Ophthalmol. 2000;84:329-31.

3. Morris DS, Selva D, Dolman PJ. Endonasal dacryocystorhinostomy in Wegener granulomatosis. Arch Ophthalmol. 2010;128:1212-4.
4. Lee BJ, Nelson CC, Lewis CD, Perry JD. External dacryocystorhinostomy surgery in patients with Wegener granulomatosis. Ophthalmic Plast Reconstr Surg. 2012;28:389-92.

5. Naeser E, Friis P, Hansen IT, Naeser K. External dacryocystorhinostomy in Wegener's granulomatosis. Acta Ophthalmol. 2013;91:776-8.

6. Verity DH, Rose GE. External dacryocystorhinostomy. In: Albert DM, Lucarelli MJ. Clinical atlas of procedures in ophthalmic and oculofacial surgery. Oxford: Oxford University Press, 2012:1021-32.

7. Kwan AS, Rose GE. Orbital Wegener's granuloma resulting from direct extension of nasal disease through a surgical rhinostomy. $\mathrm{Br}$ J Ophthalmol. 1998;82:198.

8. Rose GE. The lacrimal paradox: toward a greater understanding of success in lacrimal surgery. Ophthalmic Plast Reconstr Surg. 2004;20:262-5. 